\title{
ねじ山に集中荷重を受ける直交異方性ねじの ねじ谷底における応力分布*
}

\author{
大 滝 英 征 ${ }^{* *}$, 石 川 義 雄** \section{due to a Concentrated Load applied at its Flank} \\ The Stress Distribution at Thread Root of Anisotropic Screw Thread
}

\author{
by Hideyuki OTAKI and Yoshio ISHIKAWA
}

\begin{abstract}
The stress distribution of anisotropic mediums is hardly analyzed because of the difficulties of deriving out the stress function. Therefore, this report deals with the easy method to derive out the stress function of anisotropic mediums by using the stress function of isotropic mediums. By this method, the stress distribution at thread root of anisotropic screw thread due to a concentrated load applied at its flank is analyzed.
\end{abstract}

Key Words : Complex Stress Function, Anisotropic Mediums, Thread, Sress Distribution, Concentrated Load

\section{1. ま か き}

機械要素を異方性材料により製作し，等方性材料で は得られなかった強度, 機能を付与する研究が急速に 進展してきている. その際, 最も重要な課題の一つで ある応力分布解析については, 異方性材の場合の応力 関数の誘導がきわめて難しいこともありほとんど行わ れていない(1). 著者は, すでに, 異方性材の場合の応力 関数を等方性材料の場合の応力関数を基に誘導する簡 易手法を報告(2)したが，本報は，ぬじ谷底の応力分布 解析に適用したものである。

\section{2. 基 礎 式}

既報 ${ }^{(2)}$ で述べたように，複素関数を利用すると， $x$ ， $y$ 軸に関して直交異方性を有する材料の応力は,

$$
\begin{gathered}
\sigma_{x}+\sigma_{y}=\sigma_{u}+\sigma_{v}=\frac{4}{\dot{z}(w) \dot{\bar{z}}(\bar{w})} \frac{\partial^{2} U(w, \bar{w})}{\partial w \partial \hat{w}} \\
\cdots \cdots \cdots \cdots(1) \\
\sigma_{y}-\sigma_{x}+2 i \tau_{x y}=\frac{4}{\dot{z}(w)} \frac{\partial}{\partial w}\left[\frac{1}{\dot{z}(w)} \frac{\partial U(w, \bar{w})}{\partial w}\right]
\end{gathered}
$$

*昭和 59 年 11 月 29 日 第 929 回講演会において講演, 原稿 受付 昭和 59 年 4 月 20 日.

** 正員, 埼玉大学工学部（也338 浦和市下大久保 255).

$$
\sigma_{v}-\sigma_{u}+2 i \tau_{u v}=\frac{4}{\dot{\bar{z}}(\bar{w})}-\frac{\partial}{\partial w}\left[\frac{1}{\dot{z}(w)} \frac{\partial U(w, \bar{w})}{\partial w}\right]
$$

$$
\begin{aligned}
& \sigma_{u}, \sigma_{v}, \tau_{u v}: \text { 図 } 1 \text { に示した応力 } \\
& z(w): z \text { 平面と } w \text { 平面間の写像関数 }
\end{aligned}
$$

で求まる.

境界条件は,

$$
\left[\frac{1}{\dot{z}(w)} \frac{\partial U(w, \bar{w})}{\partial w}\right]_{\mathrm{I}}^{\mathrm{II}}=\frac{\bar{P}_{0}}{2} i
$$

$P_{0}$ : 境界上 I から II までの間に作用する外力

$$
\text { の総和 }\left(P_{0}=P_{x}+i P_{y}\right)
$$

で表される。

さて，ここで直交異方性材に関する応力関数 $U(w$, $\bar{w})$ は

$$
U(w, \bar{w})=\sum_{i=0}^{\infty} \sum_{j=0}^{\infty} U_{i j}(w, \bar{w}) \alpha_{1}^{i} \alpha_{2}^{j}
$$

$\alpha_{1}, \alpha_{2}$

$$
=\sqrt{\frac{E_{x} / G_{x y}-2 \nu_{x} \pm \sqrt{\left(E_{x} / G_{x y}-2 \nu_{x}\right)^{2}-4 E_{x} / E_{y}}}{2}}-1
$$

で表示され

$$
\begin{aligned}
& U_{00}(w, \bar{w})=\operatorname{Re}\left[\varphi_{000}(w)+\bar{z}(\bar{w}) \varphi_{001}(w)\right] \cdots(5) \\
& U_{10}(w, \bar{w})=U_{01}(w, \bar{w}) \\
& \quad=\operatorname{Re}\left[\varphi_{100}(w)+\bar{z}(\bar{w}) \varphi_{101}(w)\right. \\
& \left.-\frac{1}{4} \frac{\bar{z}(\bar{w})^{2}}{\dot{z}(w)} \dot{\varphi}_{001}(w)\right] \cdots \cdots \cdots \cdots \cdots \cdots \cdots \cdots(6)
\end{aligned}
$$




$$
\begin{aligned}
& U_{20}(w, \bar{w})=U_{02}(w, \bar{w}) \\
& \quad=\operatorname{Re}\left\{\varphi_{200}(w)+\bar{z}(\bar{w}) \varphi_{201}(w)\right. \\
& +\frac{\bar{z}(\bar{w})^{2}}{\dot{z}(w)}\left[\frac{1}{8} \dot{\varphi}_{001}(w)-\frac{1}{4} \dot{\varphi}_{101}(w)\right] \\
& \left.+\frac{\bar{z}(\bar{w})^{3}}{24} \frac{\ddot{\varphi}_{001}(w) \dot{z}(w)-\dot{\varphi}_{001}(w) \ddot{z}(w)}{\dot{z}(w)^{3}}\right\}
\end{aligned}
$$

$U_{11}(w, \bar{w})=\operatorname{Re}\left\{\varphi_{110}(w)+\bar{z}(\bar{w}) \varphi_{111}(w)+\bar{z}(\bar{w})^{2}\right.$

$$
\begin{aligned}
& \times\left[-\frac{1}{2} \frac{\dot{\varphi}_{101}(w)}{\dot{z}(w)}-\frac{1}{8}\right. \\
& \left.\times \frac{\ddot{\varphi}_{000}(w) \dot{z}(w)-\dot{\varphi}_{000}(w) \ddot{z}(w)}{\dot{z}(w)^{3}}\right] \\
& \left.+\frac{\bar{z}(\bar{w})^{3}}{24} \frac{\ddot{\varphi}_{001}(w) \dot{z}(w)-\dot{\varphi}_{001}(w) \ddot{z}(w)}{\dot{z}(w)^{3}}\right\}
\end{aligned}
$$

$$
\text { ただし，・= } \partial / \partial w
$$

の関係を有する， $U_{00}(w, \bar{w})$ は等方性材料の場合の応 力関数と同様であり，すでに多くの対象について解が 求まっているのでそれを準用すればよい. $U_{10}(w, \bar{w})$ は， $U_{00}(w, \bar{w})$ を基に境界条件を満足するような関数 形を選び求める。 $U_{20}(w, \bar{w})$ も同様，境界条件を満足 する関数形のものを $U_{00}(w, \bar{w}), U_{10}(w, \bar{w})$ を基にし て求める。このような操作を順次繰返せば, 結局直交 異方性材の応力関数が決定されることとなる。

\section{3. 直交異方性ねじのねじ谷底における応力分布}

$z$ 平面の境界 $C$ を $w$ 平面の直線境界 $\left(\zeta=u_{0}+i v\right)$ に, $z$ 平面の領域 $D$ を $w$ 平面の $\Delta$ 領域に写像する関 数として

$$
\begin{gathered}
z(w)=w-\frac{m \pi}{b} \operatorname{coth} \frac{w+a}{b} \pi \\
a, b, m: \text { 定数 }
\end{gathered}
$$

を選ぶ. $z(w)$ の画く曲線は，実際のねじ朝断面形状を 近似し, $b$ はピッチ距離と一致する。

さて，等方性材料の場合の応力関数は，すでに著者 が求めており， $U_{00}(w, \bar{w})$ は，それを準用しうる。す なわち

$$
\begin{gathered}
\varphi_{001}(w)=\frac{1}{4 \pi}\left[-P \ln \left(w-w_{0}\right)+A(w+a)^{-1}\right] \\
\dot{\varphi}_{000}(w)=\frac{1}{4 \pi}\left[-P \frac{1}{w-w_{0}}-\frac{A}{(w+a)^{2}}\right] \\
\quad \times\left[2 u_{0}-w-\frac{m \pi}{b} \operatorname{coth} \frac{2 u_{0}+a-w}{b} \pi\right] \\
-\frac{1}{4 \pi}\left[-\bar{P} \ln \left(w-w_{0}\right)+\bar{A}\left(2 u_{0}+a-w\right)^{-1}\right]
\end{gathered}
$$

$$
\times\left[1+m\left(\frac{\pi}{b}\right)^{2} \sinh ^{-2} \frac{w+a}{b} \pi\right]
$$

たたし、

$$
A=\frac{m \pi d_{0}}{b} \frac{\frac{m \pi}{b} d_{0} \tilde{b}_{0} P-\left[1+m\left(\frac{\pi}{b}\right)^{2} c_{0}\right] b_{0} \bar{P}}{\left[1+m\left(\frac{\pi}{b}\right)^{2} c_{0}\right]^{2}-\left(\frac{m \pi}{b} d_{0} h_{0}\right)^{2}}
$$

ここで, $d_{0}, b_{0}, c_{0}$ などの值は, $\dot{\varphi}_{000}(w)$ が領域 $\Delta$ 内で特異点 $w=2 u_{0}+a$ を有するので, 式(11)中の各 項を特異点まわりに級数展開し特異点を除去する際に 得られる係数である。

すなわち

$$
\begin{aligned}
& \frac{1}{w-w_{0}}=\sum_{q=0}^{\infty} b_{q}\left(w-2 u_{0}-a\right)^{q}, \\
& b_{q}=(-1)^{q}\left(2 u_{0}+a-w_{0}\right)^{-q-1} \\
& \sinh ^{-2} \frac{w+a}{b} \pi=\sum_{q=0}^{\infty} c_{q}\left(w-2 u_{0}-a\right)^{q} \\
& c_{0}=\sinh ^{-2} \frac{2\left(u_{0}+a\right)}{b} \pi \\
& c_{1}=-\frac{2 \pi}{b} \cosh \frac{2\left(u_{0}+a\right)}{b} \pi \sinh ^{-3} \frac{2\left(u_{0}+a\right)}{b} \pi \\
& \operatorname{coth}^{2} \frac{2 u_{0}+a-w}{b} \pi=\sum_{q=0}^{\infty} d_{q}\left(w-2 u_{0}-a\right)^{q-1}, \\
& d_{0}=-\frac{b}{\pi}, \quad d_{1}=0 \\
& \frac{1}{(w+a)^{2}}=\sum_{q=0}^{\infty} h_{q}\left(w-2 u_{0}-a\right)^{q}, \\
& h_{0}=\frac{1}{\left(2 u_{0}+a-w\right)^{2}}
\end{aligned}
$$

次に, $U_{10}(w, \bar{w}), U_{20}(w, \bar{w})$ などは $U_{00}(w, \bar{w})$ を 式（７）に代入したうえで，境界条件を満足させうる形 に $\varphi_{101}(w), \varphi_{100}(w)$ などを選定し, 順次決定する.い ま, Neuber が等方性材料の場合に採っている手法 ${ }^{(3)}$ と同様，境界条件式を勘案し，まず $\partial U_{10}(w, \bar{w}) / \partial w$ を求めると，

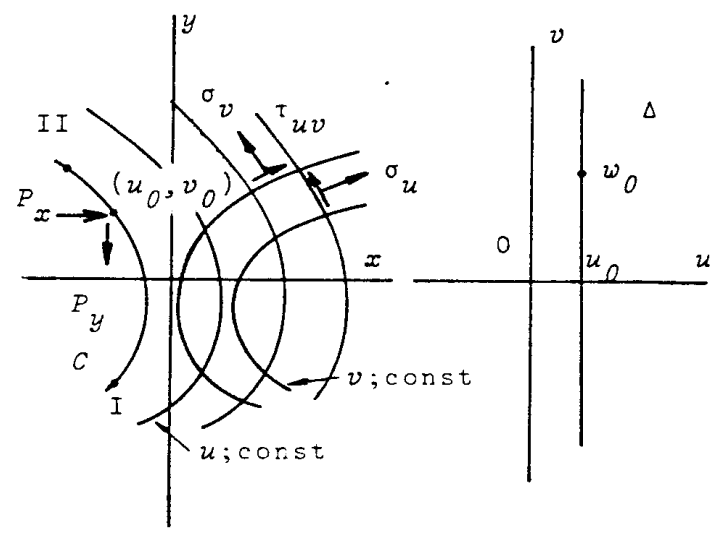
(a) $z$ 平面
(b) $u$ 平面

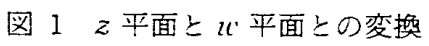




$$
\begin{aligned}
& \frac{\partial U_{10}(w, \bar{w})}{\partial w}=\dot{\varphi}_{100}(w)+\bar{z}(\bar{w}) \dot{\varphi}_{101}(w)+\dot{z}(w) \bar{\varphi}_{101}(\bar{w}) \\
& -\frac{1}{4} \bar{z}(\bar{w})^{2} \frac{\ddot{\varphi}_{001}(w) \dot{z}(w)-\dot{\varphi}_{001}(w) \ddot{z}(w)}{\dot{z}(w)^{2}} \\
& -\frac{1}{2} z(w) \dot{z}(w) \frac{\frac{\dot{\varphi_{001}}}{\bar{w}^{\prime}}(\bar{w})}{\dot{\bar{z}}(\bar{w})}
\end{aligned}
$$

ここて

$$
\varphi_{101}(w)=-\frac{1}{4 \pi} \ln \left(w-w_{0}\right)+\frac{B}{w+a}+\frac{C}{(w+a)^{2}}-\frac{1}{2} z(w) \frac{\dot{\varphi}_{001}(w)}{\dot{z}(w)}
$$

とする. 式(13)の右辺第 3 項は，式(10)を勘案したうえで選定した項であり，第 4 項は無限遠点で応力が零に収束 するように選定した項である。

いま，境界 $u=u_{0}\left(\bar{\zeta}=2 u_{0}-\zeta\right) に て$

$$
\left.\begin{array}{l}
\left.\frac{1}{\dot{z}(w)} \frac{\partial U_{10}(w, \bar{w})}{\partial w}\right|_{w=s}=\mu \\
\mu=\frac{\bar{P}}{4 \pi} \ln (-1)
\end{array}\right\}
$$

を満足するものとして，式(12)を整理すると

$$
\begin{aligned}
\dot{\varphi}_{100} & (w)=-\left(2 u_{0}-w-\frac{m \pi}{b} \operatorname{coth} \frac{2 u_{0}+a-w}{b} \pi\right)\left\{-\frac{P}{4 \pi} \frac{1}{w-w_{0}}-\frac{B}{(w+a)^{2}}-\frac{2 C}{(w+a)^{3}}\right. \\
& \left.-\frac{1}{2}\left[\dot{\varphi}_{001}(w)+z(w) \frac{\ddot{\varphi}_{001}(w) \dot{z}(w)-\dot{\varphi}_{001}(w) \ddot{z}(w)}{\dot{z}(w)^{2}}\right]\right\} \\
& -\dot{z}(w)\left\{-\frac{\bar{P}}{4 \pi} \ln \left(w-w_{0}\right)+\frac{\bar{B}}{2 u_{0}+a-w}+\frac{\bar{C}}{\left(2 u_{0}+a-w\right)^{2}}+\frac{1}{8 \pi}\right. \\
& \left.\times\left(2 u_{0}-w-\frac{m \pi}{b} \operatorname{coth} \frac{2 u_{0}+a-w}{2} \pi\right)\left[\frac{\bar{P}}{w_{0}-w}+\frac{\bar{A}}{\left(2 u_{0}+a-w\right)^{2}}\right] /\left[1+m\left(\frac{\pi}{b}\right)^{2} \sinh ^{-2} \frac{2 u_{0}+a-w}{b} \pi\right]\right\} \\
& +\frac{1}{4}\left(2 u_{0}-w-\frac{m \pi}{b} \operatorname{coth} \frac{2 u_{0}+a-w}{b} \pi\right)^{2} \frac{\ddot{\varphi}_{001}(w) \dot{z}(w)-\dot{\varphi}_{001}(w) \ddot{z}(w)}{\dot{z}(w)^{2}} \\
& \left.-\frac{1}{8 \pi} z(w) \dot{z}(w)\left[\frac{\bar{P}}{w_{0}-w}+\frac{\bar{A}}{\left(2 u_{0}+a-w\right)^{2}}\right] /\left[1+m\left(\frac{\pi}{b}\right)^{2} \sinh ^{-2} \frac{2 u_{0}+a-w}{b} \pi\right] \ldots \ldots \ldots \ldots \ldots . . . . . . . .15\right)
\end{aligned}
$$

$\dot{\varphi}_{100}(w)$ は, 領域 $\Delta$ 内で特異点を有してはならぬが, 式(15)は $w=2 u_{0}+a に て$ 特異点を有する，そこで，これを除 去するため，まず $\dot{\varphi}_{100}(w)$ に含まれる各項を特異点まわりにローラン級数展開し

$$
\begin{aligned}
& \frac{1}{(w+a)^{3}}=\sum_{q=0}^{\infty} l_{q}\left(w-2 u_{0}-a\right)^{q}, \quad l_{0}=\frac{1}{\left[2\left(u_{0}+a\right)\right]^{3}}, \quad l_{1}=-\frac{3}{\left[2\left(u_{0}+a\right)\right]^{4}} \\
& \operatorname{coth}^{2} \frac{2 u_{0}+a-w}{b} \pi=\sum_{q=0}^{\infty} s_{q}\left(w-2 u_{0}-a\right)^{q-2}, \quad s_{0}=\left(\frac{b}{\pi}\right)^{2}, \quad s_{1}=0, \quad s_{2}=\frac{1}{3} \\
& \frac{1}{\left(w-w_{0}\right)^{2}}=\sum_{q=0}^{\infty} i_{q}\left(w-2 u_{0}-a\right)^{q}, \quad i_{0}=\frac{1}{\left(2 u_{0}+a-w\right)^{2}}, \quad i_{1}=-\frac{2}{\left(2 u_{0}+a-w\right)^{3}} \\
& \operatorname{coth} \frac{w+a}{b} \pi=\sum_{q=0}^{\infty} g_{q}\left(w-2 u_{0}-a\right)^{q}, \quad g_{0}=\operatorname{coth} \frac{2\left(u_{0}+a\right)}{b} \pi, \quad g_{1}=-\frac{\pi}{b} \sinh ^{-2} \frac{2\left(u_{0}+a\right)}{b} \pi \\
& \dot{\varphi}_{001}(w)+z(w) \frac{\ddot{\varphi}_{001}(w)-\dot{\varphi}_{001}(w) \ddot{z}(w)}{\dot{z}(w)^{2}}=\sum_{q=0}^{\infty} f_{a q}\left(w-2 u_{0}-a\right)^{q} \\
& f_{a q}=\left.\frac{1}{q !} \frac{\mathrm{d}^{q}}{\mathrm{~d} w^{q}}\left[\dot{\varphi}_{001}(w)+z(w) \frac{\ddot{\varphi}_{001}(w) \dot{z}(w)-\dot{\varphi}_{001}(w) \ddot{z}(w)}{\dot{z}(w)^{2}}\right]\right|_{w=2 u_{0}+a} \\
& \frac{1}{4 \pi}\left[-\frac{\bar{P}}{w_{0}-w}-\frac{\bar{A}}{\left(2 u_{0}+a-w\right)^{2}}\right] /\left[1+m\left(\frac{\pi}{b}\right)^{2} \sinh ^{-2} \frac{2 u_{0}+a-w}{b} \pi=\sum_{q=0}^{\infty} q q\left(w-2 u_{0}-a\right)^{q}\right. \\
& q_{0}=-\frac{1}{4 \pi} \frac{\bar{A}}{m} \\
& {\left[1+m\left(\frac{\pi}{b}\right)^{2} \sinh ^{-2} \frac{w+a}{b} \pi\right]^{-2}=\sum_{q=0}^{\infty} t_{q}\left(w-2 U_{0}-a\right)^{q}, \quad t_{0}=\left[1+m\left(\frac{\pi}{b}\right)^{2} \sinh ^{-2} \frac{2\left(u_{0}+a\right)}{b} \pi\right]^{-2}}
\end{aligned}
$$


を得る.式(16)を式(15)に代入し, 特異点を有する項を選び出すと

$\left(w-2 u_{0}-a\right)^{-1}$ の項より,

$$
\begin{aligned}
& -\frac{m \pi}{b} d_{0}\left(-\frac{P}{4 \pi} b_{0}-B h_{0}-2 C l_{0}-\frac{1}{2} f_{00}\right)-\left[1+m\left(\frac{\pi}{b}\right)^{2} c_{0}\right]\left(\bar{B}-\frac{q_{0}}{2} \frac{m \pi d_{0}}{b}\right)+m\left(\frac{\pi}{b}\right)^{2} c_{1} \bar{C} \\
& -\frac{1}{16 \pi}\left(\frac{m \pi}{b}\right)^{2} s_{0}\left[t_{1}\left\{\left(P i_{0}+2 A l_{0}\right)\left[1+m\left(\frac{\pi}{b}\right)^{2} c_{0}\right]-2 m\left(\frac{\pi}{b}\right)^{3} c_{0} g_{0}\left(P b_{0}+A h_{0}\right)\right\}\right. \\
& +t_{0}\left\{\left(P i_{1}+2 A l_{1}\right)\left[1+m\left(\frac{\pi}{b}\right)^{2} c_{0}\right]+\left(P i_{0}+2 A l_{0}\right) m\left(\frac{\pi}{b}\right)^{2} c_{1}-2 m\left(\frac{\pi}{b}\right)^{3} C_{0} g_{0}\left(P b_{1}+A h_{1}\right)\right. \\
& \left.\left.-2 m\left(\frac{\pi}{b}\right)^{3}\left(c_{0} g_{1}+c_{1} g_{0}\right)\left(P b_{0}+A h_{0}\right)\right\}\right]-\frac{1}{8 \pi} \frac{a m \pi d_{0} t_{0}}{b}\left\{\left(P i_{0}+2 A l_{0}\right)\left[1+m\left(\frac{\pi}{b}\right)^{2} c_{0}\right]\right. \\
& \left.-2 m\left(\frac{\pi}{b}\right)^{3} c_{0} g_{0}\left(P b_{0}+A h_{0}\right)\right\}-\frac{1}{16 \pi}\left(\frac{m \pi}{b}\right)^{2} s_{1} t_{0}\left\{\left(P i_{0}+2 A l_{0}\right)\left[1+m\left(\frac{\pi}{b}\right)^{2} c_{0}\right]\right. \\
& \left.-2 m\left(\frac{\pi}{b}\right)^{3} c_{0} g_{0}\left(P b_{0}+A h_{0}\right)\right\}=0
\end{aligned}
$$

$\left(w-2 u_{0}-a\right)^{-2}$ の項より,

$$
\begin{aligned}
& {\left[1+m\left(\frac{\pi}{b}\right)^{2} c_{0}\right] \bar{C}-\frac{1}{16 \pi}\left(\frac{m \pi}{b}\right)^{2} s_{0} t_{0}\left\{\left(P i_{0}+2 A l_{0}\right)\left[1+m\left(\frac{\pi}{b}\right)^{2} c_{0}\right]\right.} \\
& \left.-2 m\left(\frac{\pi}{b}\right)^{3} c_{0} g_{0}\left(P b_{0}+A h_{0}\right)\right\}=0
\end{aligned}
$$

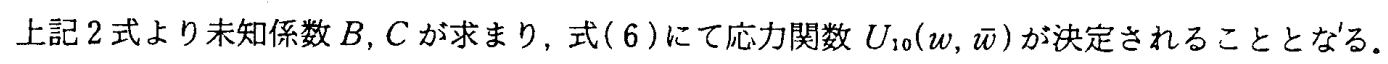

さてここで, 式（6）を境界条件式（3）に代入し，かつI，IIを自由境界上の任意点にとると，確かに

$$
\left[\frac{1}{\dot{z}(w)} \frac{\partial U_{10}(w, \bar{w})}{\partial w}\right]_{1}^{11}=0
$$

となる. 荷重点を領域に I， II をとると

$$
\left[\frac{1}{\dot{z}(w)} \frac{\partial U_{10}(w, \bar{w})}{\partial w}\right]_{1}^{\mathrm{II}}=\left[\frac{\bar{P}}{4 \pi} \ln \frac{\bar{w}-\bar{w}_{0}}{w-w_{0}}\right]_{1}^{\mathrm{II}}=\left.\frac{\bar{P}}{4 \pi}\left[\ln \frac{1-i\left(v-v_{0}\right) /\left(u-u_{0}\right)}{1+i\left(v-v_{0}\right) /\left(u-u_{0}\right)}\right]_{1}^{\mathrm{II}}\right|_{u=u_{0}}
$$

となるが,ここで関数論で周知の

$$
\ln \frac{1-i\left(v-v_{0}\right) /\left(u-u_{0}\right)}{1+i\left(v-v_{0}\right) /\left(u-u_{0}\right)}=-2 i \operatorname{arct} t_{0}\left|\frac{v-v_{0}}{u-u_{0}}\right|
$$

なる関係を利用し, かつ $u=u_{0}$ を加味すると

$$
\left[\frac{1}{\dot{z}(w)} \frac{\partial U_{10}(w, \bar{w})}{\partial w}\right]_{1}^{11}=\frac{\bar{P}}{2} i
$$

となり，境界条件を満足する式形が得られる，P と $P_{0}$ との関係は後述する.

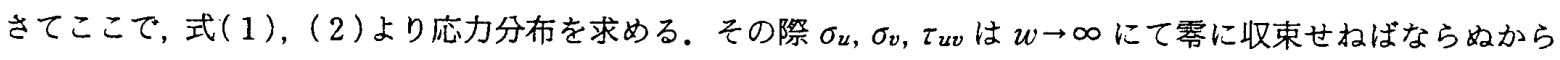

$$
\left\{\begin{array}{l}
\left.\frac{4}{\dot{z}(w) \dot{\bar{z}}(\bar{w})} \frac{\partial^{2} U_{10}(w, \bar{w})}{\partial w \partial w}\right|_{w-\infty}=0 \ldots \ldots . . \\
\left.\frac{4}{\dot{\bar{z}}(\bar{w})} \frac{\partial}{\partial w}\left[\frac{1}{\dot{z}(w)} \frac{\partial U_{10}(w, \bar{w})}{\partial w}\right]\right|_{w-\infty}=0
\end{array}\right.
$$

でなくてはならぬ.ちなみに

$$
\begin{aligned}
& \frac{4}{\dot{z}(w) \dot{\bar{z}}(\bar{w})} \frac{\partial^{2} U_{10}(w, \bar{w})}{\partial w \partial \bar{w}}=\operatorname{Re}\left\{\frac{1}{\dot{z}(w)}\left[-\frac{P}{4 \pi} \frac{1}{w-w_{0}}-\frac{B}{(w+a)^{2}}-\frac{2 C}{(w+a)^{3}}-\frac{1}{2} \dot{\varphi}_{001}(w)\right]\right. \\
& \left.-\frac{1}{2}[z(w)+\bar{z}(\bar{w})] \frac{\ddot{\varphi}_{001}(w) \dot{z}(w)-\ddot{\varphi}_{001}(w) \ddot{z}(w)}{\dot{z}(w)^{3}}\right\} \ldots \ldots \ldots \ldots \ldots \ldots \ldots \ldots \ldots \ldots \ldots \ldots \ldots \ldots \ldots \ldots \ldots \ldots \ldots \ldots \ldots \ldots \ldots \ldots \ldots \ldots \ldots \ldots \ldots \ldots \ldots \ldots \ldots
\end{aligned}
$$

ただし, $\dot{\varphi}_{001}(w)=\frac{1}{4 \pi}\left[-\frac{P}{w-w_{0}}-\frac{A}{(w+a)^{2}}\right]$

$$
\ddot{\varphi}_{001}(w)=\frac{1}{4 \pi}\left[\frac{P}{\left(w-w_{0}\right)^{2}}+\frac{2 A}{(w+a)^{3}}\right]
$$

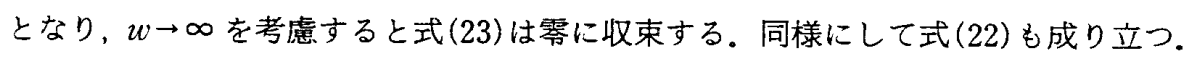

次に同様にして， $U_{20}(w, \bar{w})$ を求める. 式 $(7)$ より，境界条件式を勘案して $\partial U_{20}(u, \bar{w}) / \partial w$ を求めると， 


$$
\begin{aligned}
& \frac{\partial U_{20}(w, \bar{w})}{\partial w}=\dot{\varphi}_{200}(w)+\bar{z}(\bar{w}) \dot{\varphi}_{201}(w)+\dot{z}(w) \bar{\varphi}_{201}(\bar{w})-\frac{\bar{z}(\bar{w})^{2}}{4} \frac{\ddot{\varphi}_{101}(w) \dot{z}(w)-\dot{\varphi}_{101}(w) \ddot{z}(w)}{\dot{z}(w)^{2}} \\
& \quad-\frac{\dot{z}(w) z(w)}{2} \frac{\dot{\bar{\varphi}}_{101}(\bar{w})}{\dot{\bar{z}}(\bar{w})}+\frac{\bar{z}(\bar{w})^{2}}{8} \frac{\ddot{\varphi}_{001}(w) \dot{z}(w)-\dot{\varphi}_{001}(w) \ddot{z}(w)}{\dot{z}(w)^{2}}+\frac{\dot{z}(w) z(w)}{4} \frac{\dot{\bar{\varphi}}_{001}(\bar{w})}{\dot{\bar{z}}(\bar{w})} \\
& \quad+\frac{1}{24} \frac{\left[\ddot{\varphi}_{001}(w) \dot{z}(w)-\dot{\varphi}_{001}(w) \ddot{z}(w)\right] \dot{z}(w)-3\left[\ddot{\varphi}_{001}(w) \dot{z}(w)-\dot{\varphi}_{001}(w) \ddot{z}(w)\right] \ddot{z}(w)}{\dot{z}(w)^{4}} \\
& \quad+\frac{z(w)^{2} \dot{z}(w)}{8} \frac{\ddot{\bar{\varphi}}_{001}(\bar{w}) \dot{\bar{z}}(\bar{w})-\dot{\bar{\varphi}}_{001}(\bar{w}) \ddot{z}(\bar{w})}{\dot{\bar{z}}(\bar{w})^{3}} \ldots \ldots \ldots \ldots \ldots \ldots \ldots \ldots \ldots \ldots \ldots \ldots \ldots \ldots \ldots \ldots \ldots \ldots \ldots \ldots \ldots \ldots \ldots \ldots \ldots \ldots \ldots \ldots \ldots \ldots \ldots \ldots \ldots
\end{aligned}
$$

ここで, $\varphi_{101}(w), \varphi_{001}(w)$ を勘案したうえで

$$
\begin{gathered}
\varphi_{201}(w)=-\frac{P}{4 \pi} \ln \left(w-w_{0}\right)+\frac{D}{w+a}+\frac{E}{(w+a)^{2}}+\frac{F}{(w+a)^{3}}-\frac{1}{2} z(w) \frac{\dot{\varphi}_{101}(w)}{\dot{z}(w)} \\
+\frac{1}{4} z(w) \frac{\dot{\varphi}_{001}(w)}{\dot{z}(w)}-\frac{1}{8} z(w)^{2} \frac{\ddot{\varphi}_{001} \dot{z}(w)-\dot{\varphi}_{001}(w) \ddot{z}(w)}{\dot{z}(w)^{3}}
\end{gathered}
$$

とし、さらに境界ちにて

$$
\left.\frac{1}{\dot{z}(w)} \frac{\partial U_{20}(w, \bar{w})}{\partial w}\right|_{w=\zeta}=\mu, \quad \mu=\frac{\bar{P}}{4 \pi} \ln (-1)
$$

を満足するものとしたうえで, 式 $(24) を \dot{\varphi}_{200}(w)$ にて整理する.すると, $\dot{\varphi}_{200}(w)$ は $\dot{\varphi}_{100}(w)$ の場合と同様, 領域 $\Delta$ 内の $w=2 u_{0}+a$ にて特異点を有するので, これを除去する必要性がある.そこで, $\dot{\varphi}_{200}(w)$ に含まれる各項を特異 点まわりに級数展開すると

$$
\begin{aligned}
& \frac{\ddot{\varphi}_{101}(w) \dot{z}(w)-\dot{\varphi}_{101}(w) \ddot{z}(w)}{\dot{z}(w)^{2}}=\sum_{q=0}^{\infty} a_{a q}\left(w-2 u_{0}-a\right)^{q} \\
& a_{a q}=\left.\frac{1}{q !} \frac{\mathrm{d}^{q}}{\mathrm{~d} w^{q}}\left[\frac{\ddot{\varphi}_{101}(w) \dot{z}(w)-\dot{\varphi}_{101}(w) \ddot{z}(w)}{\dot{z}(w)^{2}}\right]\right|_{w=2 u_{0}+a} \\
& \frac{\ddot{\varphi}_{001}(w) \dot{z}(w)-\dot{\varphi}_{001}(w) \ddot{z}(w)}{\dot{z}(w)^{2}}=\sum_{q=0}^{\infty} b_{a q}\left(w-2 u_{0}-a\right)^{q} \\
& b_{a q}=\left.\frac{1}{q !} \frac{\mathrm{d}^{q}}{\mathrm{~d} w^{q}}\left[\frac{\ddot{\varphi}_{001}(w) \dot{z}(w)-\dot{\varphi}_{001}(w) \ddot{z}(w)}{\dot{z}(w)^{2}}\right]\right|_{w=2 u_{0}+a} \\
& \frac{\left[\ddot{\varphi}_{001}(w) \dot{z}(w)-\dot{\varphi}_{001}(w) \ddot{z}(w)\right] \dot{z}(w)-3\left[\ddot{\varphi}_{001}(w) \dot{z}(w)-\dot{\varphi}_{001}(w) \ddot{z}(w)\right]}{\dot{z}(w)^{4}} \\
& =\sum_{q=0}^{\infty} c_{a q}\left(w-2 u_{0}-a\right)^{q} \\
& c_{a q}=\left.\frac{1}{q !} \frac{\mathrm{d}^{q}}{\mathrm{~d} w^{q}}\left\{\frac{\left[\dot{\varphi}_{001}(w) \dot{z}(w)-\ddot{\varphi}_{001}(w) \ddot{z}(w)\right] \dot{z}(w)-3\left[\ddot{\varphi}_{001}(w) \dot{z}(w)-\dot{\varphi}_{001}(w) \ddot{z}(w)\right]}{\dot{z}(w)^{4}}\right\}\right|_{w=2 u_{0}+a} \\
& \bar{z}(\bar{w}) \frac{\dot{\bar{\varphi}}_{101}(\bar{w})}{\dot{\bar{z}}(\bar{w})}=\sum_{q=0}^{\infty} d_{a q}\left(w-2 u_{0}-a\right)^{q-2} \\
& d_{a 0}=2 \bar{C}, \quad d_{a 1}=\bar{B}-\bar{A} / 8 \pi \\
& \frac{\dot{\bar{\varphi}}_{101}(\bar{w})}{\dot{\bar{z}}(\bar{w})}=\sum_{q=0}^{\infty} e_{a q}\left(w-2 u_{0}-a\right)^{q-1} \\
& e_{a 0}=2 \bar{C} / m \\
& \bar{z}(\bar{w})^{2} \frac{\ddot{\bar{\varphi}}_{001}(\bar{w}) \dot{\bar{z}}(w)-\dot{\bar{\varphi}}_{001}(\bar{w}) \ddot{\bar{z}}(\bar{w})}{\dot{\bar{z}}(\bar{w})^{3}}=\sum_{q=0}^{\infty} g_{a q}\left(w-2 u_{0}-a\right)^{q-1} \\
& g_{a 0}=0 \\
& \bar{z}(\bar{w}) \frac{\dot{\bar{\varphi}}_{001}(\bar{w})}{\overline{\bar{z}}(\bar{w})}=\sum_{q=0}^{\infty} h_{a q}\left(w-2 u_{0}-a\right)^{q-1} \\
& h_{a 0}=-\bar{A} / 4 \pi \\
& -\frac{1}{2} \dot{\varphi}_{101}(w)+\frac{1}{4} \dot{\varphi}_{001}(w)-\frac{1}{2} z(w) \frac{\ddot{\varphi}_{101}(w) \dot{z}(w)-\dot{\varphi}_{101}(w) \ddot{z}(w)}{\dot{z}(w)^{2}} \\
& -\frac{1}{8} z(w)^{2} \frac{\left[\dddot{\varphi}_{001}(w) \dot{z}(w)-\dot{\varphi}_{001}(w) z(w)\right] \dot{z}(w)-3\left[\ddot{\varphi}_{001}(w) \dot{z}(w)-\dot{\varphi}_{001}(w) \ddot{z}(w)\right] \ddot{z}(w)}{\dot{z}(w)^{4}}
\end{aligned}
$$




$$
\begin{aligned}
&=\sum_{q=0}^{\infty} l_{a q}\left(w-2 u_{0}-a\right)^{q} \\
& l_{a q}=\frac{1}{q !} \frac{\mathrm{d}^{q}}{\mathrm{~d} w^{q}}\left\{-\frac{1}{2} \dot{\varphi}_{101}(w)+\frac{1}{4} \dot{\varphi}_{001}(w)-\frac{z(w)}{2} \frac{\ddot{\varphi}_{101}(w) \dot{z}(w)-\dot{\varphi}_{101}(w) \ddot{z}(w)}{\dot{z}(w)^{2}}\right. \\
&\left.-\frac{z(w)^{2}}{8} \frac{\left[\varphi_{001}(w) \dot{z}(w)-\dot{\varphi}_{001}(w) \dddot{z}(w)\right] \dot{z}(w)-3\left[\ddot{\varphi}_{001}(w) \dot{z}(w)-\dot{\varphi}_{001}(w) \ddot{z}(w)\right] \ddot{z}(w)}{\dot{z}(w)^{4}}\right\}\left.\right|_{w=2 u_{0}+a} \\
& \operatorname{coth}^{3} \frac{2 u_{0}+a-w}{b}=\sum_{q=0}^{\infty} v_{a q}\left(w-2 u_{0}-a\right)^{q-3}
\end{aligned}
$$$$
v_{a 0}=(-b / \pi)^{3}, \quad v_{a 1}=0, \quad v_{a 2}=-b \pi / 2 \text { ! }
$$

となる。いま式(27)を式(24)に代入， $\dot{\varphi}_{200}(w)$ につて整理したうえで特異点を有する項を選び出すと，(wー2 $u_{0}$ -a) $)^{-1}$ の項より,

$$
\left.\begin{array}{l}
-\frac{m \pi}{b} d_{0}\left(-\frac{P}{4 \pi} b_{0}-D h_{0}-2 E l_{0}-3 F m_{0}+l_{a 0}\right)+\left[1+m\left(\frac{\pi}{b}\right)^{2} c_{0}\right]\left(-\bar{D}+\frac{1}{4} h_{a 0}-\frac{1}{2} d_{a 0}-\frac{1}{8} g_{a 0}\right) \\
+m\left(\frac{\pi}{b}\right)^{2} c_{1} \bar{E}-m\left(\frac{\pi}{b}\right)^{2} c_{2} \bar{F}-\frac{1}{2} d_{a 0} m\left(\frac{\pi}{b}\right)^{2} c_{1}-\frac{1}{4}\left[\left(\frac{m \pi}{b}\right)^{2}\left(s_{0} a_{a 1}+s_{1} a_{a 0}\right)+\frac{2 m \pi}{b} a d_{0} a_{a 0}\right] \\
-\frac{1}{2}\left[1+m\left(\frac{\pi}{b}\right)^{2} c_{0}\right]\left(2 u_{0}+a-\frac{m \pi}{b} g_{0}\right) c_{a 0}+\frac{1}{8}\left[\left(\frac{m \pi}{b}\right)^{2}\left(s_{0} b_{a 1}+s_{1} b_{a 0}\right)+\frac{2 m \pi}{b} a d_{0} b_{a 0}\right] \\
+\frac{1}{24}\left[-3 a^{2} \frac{m \pi}{b} d_{0} c_{a 0}-3 a\left(\frac{m \pi}{b}\right)^{2} s_{0} c_{a 1}-3 a\left(\frac{m \pi}{b}\right)^{2} s_{1} c_{a 0}-3\left(\frac{m \pi}{b}\right)^{2} s_{0} c_{a 0}\right. \\
\left.-\left(\frac{m \pi}{b}\right)^{3}\left(v_{a 0} c_{a 2}+v_{a 1} c_{a 1}+v_{a 2} c_{a 0}\right)\right]=0
\end{array}\right\}
$$

$\left(w-2 u_{0}-a\right)^{-2}$ の項より,

$$
\begin{aligned}
& {\left[1+m\left(\frac{\pi}{b}\right)^{2} c_{0}\right]\left(\bar{E}-\frac{1}{2} d_{a 0}\right)-m\left(\frac{\pi}{b}\right)^{2} c_{1} \bar{F}-\frac{1}{4}\left(\frac{m \pi}{b}\right)^{2} s_{0} a_{a 0} } \\
& +\frac{1}{8}\left(\frac{m \pi}{b}\right)^{2} s_{0} b_{a 0}+\frac{1}{24}\left[-3\left(\frac{m \pi}{b}\right)^{2} a s_{0} C_{a 0}-\left(\frac{m \pi}{b}\right)^{3}\left(v_{a 1} c_{a 0}\right.\right. \\
\left(w-2 u_{0}-a\right)^{-3} \text { の項より, } & -\left[1+m\left(\frac{\pi}{b}\right)^{2} c_{0}\right] \bar{F}-\frac{1}{24}\left(\frac{m \pi}{b}\right)^{3} v_{a 0} c_{a 0}=0
\end{aligned}
$$$$
+\frac{1}{8}\left(\frac{m \pi}{b}\right)^{2} s_{0} b_{a 0}+\frac{1}{24}\left[-3\left(\frac{m \pi}{b}\right)^{2} a s_{0} C_{a 0}-\left(\frac{m \pi}{b}\right)^{3}\left(v_{a 1} C_{a 0}+v_{a 0} C_{a 1}\right)\right]=0
$$

以上の連立一次方程式を解けば末知係数 $D, E, F$ が

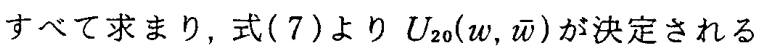

こととなる.

ここで，境界条件式 ( 3 )にて，I，IIを自由境界上 の任意点に選ぶと, 確かに

$$
\left[\frac{1}{\dot{z}(w)} \frac{\partial U_{20}(w, \bar{w})}{\partial w}\right]_{1}^{11}=0
$$

となり，また荷重点を含む領域に選ぶと，

$$
\left[\frac{1}{\dot{z}(w)} \frac{\partial U_{20}(w, \bar{w})}{\partial w}\right]_{1}^{\mathrm{II}}=\left[\frac{\bar{P}}{4 \pi} \ln \frac{\bar{w}-\bar{w}_{0}}{w-w_{0}}\right]_{\mathrm{I}}^{\mathrm{II}}=\frac{\bar{P}}{2} i
$$

となり境界条件を満足する式形となる。

次に, $U_{20}(w, \bar{w})$ にて計算される応力の無限遠点に おける值を $U_{10}(w, \bar{w})$ の場合と同様にして検討して みると確かに

$$
\left\{\begin{array}{l}
\left.\frac{4}{\dot{z}(w) \dot{\bar{z}}(\bar{w})} \frac{\partial^{2} U_{20}(w, \bar{w})}{\partial w \partial \bar{w}}\right|_{w-\infty}=0 \\
\left.\frac{4}{\dot{\bar{z}}(\bar{w})} \frac{\partial}{\partial w}\left[\frac{1}{\dot{z}(w)} \frac{\partial U_{20}(w, \bar{w})}{\partial w}\right]\right|_{w-\infty}=0
\end{array}\right.
$$

を満足し, 無限遠点にて応力が零に収束するという条 件を満足している。

以上より, $U_{20}(w, \bar{w})$ も適合条件式, 境界条件式を 満足することがわかる。 $U_{02}(w, \bar{w}) に$ 関しても全く同 様にして求まり $U_{02}(w, \bar{w})=U_{20}(w, \bar{w})$ の関係があ る.

さて,ここで $P$ と $P_{0}$ との関係について䢡べてお く.いま仮に, $U_{00}(w, \bar{w}), U_{10}(w, \bar{w}), U_{01}(w, \bar{w})$, $U_{20}(w, \bar{w}), U_{02}(w, \bar{w})$ までの項を取って応力解析す る場合は，境界条件式 ( 3 )，(20)，(29）を考慮すると

$$
P=P_{0} /\left(1+\alpha_{1}+\alpha_{2}+\alpha_{1}^{2}+\alpha_{2}^{2}\right)
$$

なる関係を有することがわかる。

以上のようにして, 順次 $U_{i j}(w, \bar{w})$ を求めていけ ば, 直交異方性材の場合の応力関数が式 (4)により決 定されることとなる，直交異方性材では，一般に $\left|\alpha_{3}\right|$, $\left|\alpha_{2}\right|<1$ となるので $\alpha_{1}^{i} \alpha_{2}^{j}$ の值を加味すると, あまり多 くの項まで取らなくても近似的には応力分布を求めう ると考えられる。

図 2,3 は, 一例として $U_{00}(w, \bar{w}) \sim U_{02}(w, \bar{w}) の$ 


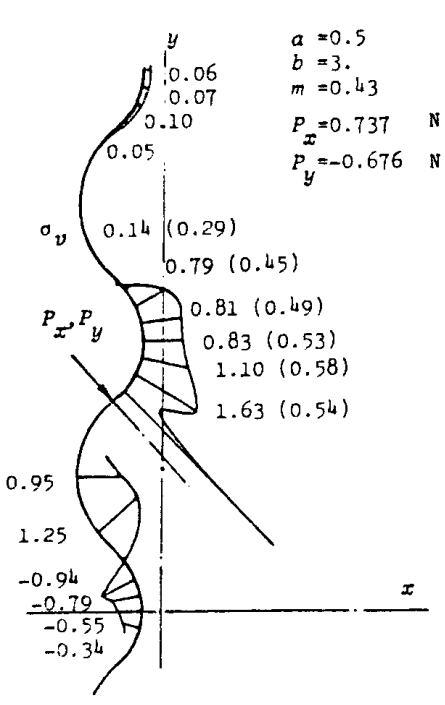

(a)

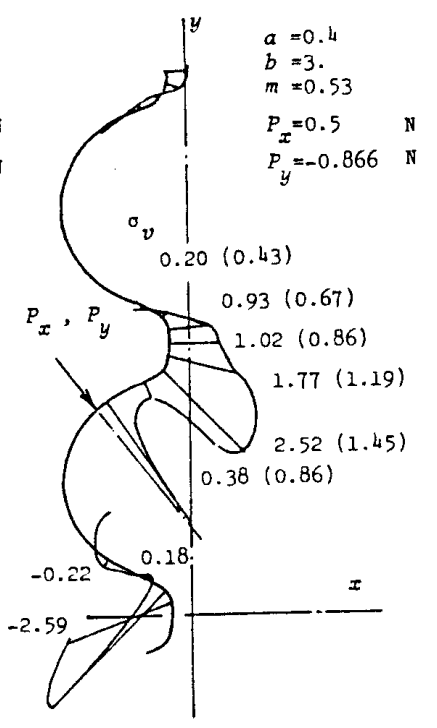

(b)

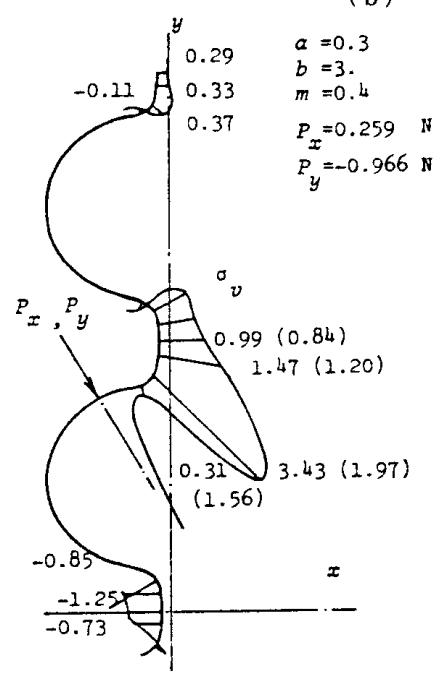

(c)

（）内：等方性材の場合

图 2 ねじ谷底の応力分布 $\sigma v\left(E_{x}=10 \mathrm{GPa}, E_{y}=40 \mathrm{GPa}\right.$, $G_{x y}=8\left(\mathrm{PPa}, \nu_{x}=0.075\right)$

項を取って計算した各種ねじ谷底における応力分布を 示したものである. 図 2,3 上り， $x, y$ 軸方向の剛性に よりるじ谷底に生じる応力分布が異なり，ねじ谷底の

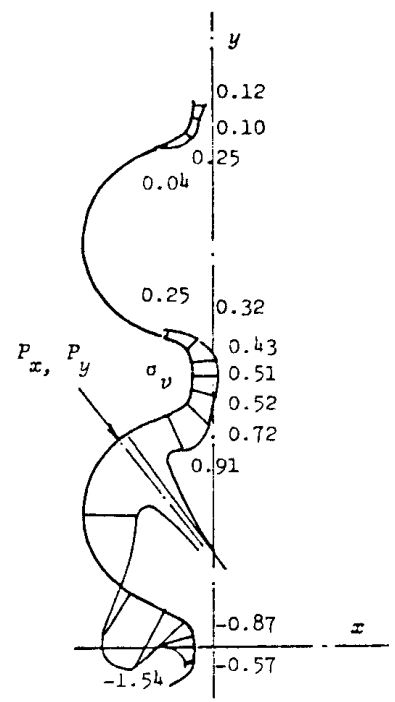

(a)

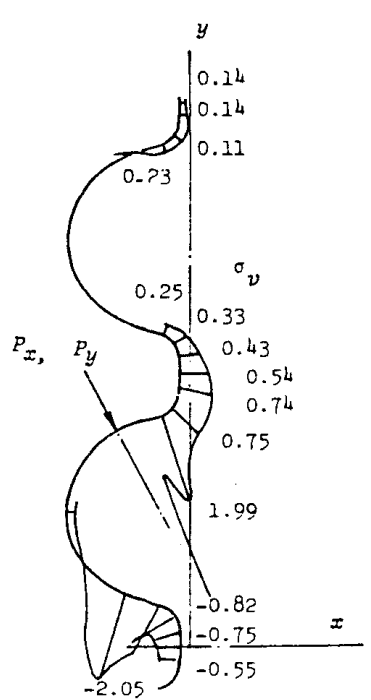

(b)
図 3 ねじ谷底の応力分布 $\sigma_{v} \mathrm{MPa}(E x=40 \mathrm{GPa}$, $\left.E_{y}=10 \mathrm{GPa}, G_{x y}=8 \mathrm{GPa}, \nu_{x}=0.3\right)$

丸み半径が比較的大きい範囲では, $x$ 軸方向の剛性が 高い場合のほうが発生する最大応力は小さくなるよう である、また，いずれの場合も，等方性の場合に比し 最大応力は小さくなる．以上のように，本手法によれ ば，材料の剛性，枕じの幾何学的形状等の応力分布へ 及ほす影響の把握が容易となる。

\section{4. むす ひ}

本報では，直交異方性ねじの応力関数を誘道した， この応力関数を利用すれば，瀻維強化複合材料製ねじ の标じ谷底における応力分布も把握できることとな り，織維配列などの最適化に資することとなる。

$$
\text { 文献 }
$$

（1）佐藤，直交異方性体問題の新解法，(昭 49)，90，現代工 学社.

（2）大滝, 北陸信越支部講演会，No. 847-1 (昭 59-9)，28.

(3) Neuber, H., Z. Angew. Math. Mech., 44-7 (1964), 285.

\section{討論}

\section{〔質問〕田中道 彦〔信州大学工学部〕} ねじの応力解析の分野で，貴重な研究を数多くなさ っておられることに敬意を表する。

（1） $U_{00}$ をもとに $U_{i j}$ を算出していく方法は, 等 方性材料での解からの捸動量を補正するように逐次 $U_{i j}$ を求めていくものと思われる. 図 2 の応力分布を みると，等方性解との差のかなり大きい部分もある が, $U_{02}, U_{20}$ で打切っても良好か。
（2） $U_{00} \sim U_{02}$ までの結果を紹介してあるが， $\alpha_{1}$, $\alpha_{2}$ から考えて， $U_{02}, U_{20}$ と $U_{11}$ は同程度のレベルで はないかとも思われるが， $U_{11}$ の影響は小さいと考え るか。

（3）荷重 $\left(P_{x}, P_{y}\right)$ の作用点付近では応力が乱れ, このことは図 2，3でも明らかになっている．実際の 対じ山接触面はねじ谷底近くまで広がっているが，外 力分布の程度は谷底応力に影響を及ぼすと考えられる 
か.

（回答（1）本解析では, $i, j$ の項数が多くな ると，応力関数 $U_{i j}$ の誘道も繁雜となり難しくなる. しかし，幸いにも本写像関数で与えられる形状では， $U_{00}, U_{10}, U_{20}$ の值のオーダは同程度である一方, $\alpha_{1}^{1} \alpha_{2}^{j}$ の值が $i, j$ の項数の增加とともに極めて小さく なる。そ机ゆえ， $i+j \simeq 2$ 程度まで取れば近似的に応 力分布は求め得ると考えたわけである。しかし，これ も写像関数により与えられる形状によっては，さらに 項数を増す必要性があるものと考える。

（2）ご指摘のように本写像関数で与えられる形状 に対しての $U_{i j(i, j=0 \sim 2)}$ の值は同程度内レべルにある. 一方， $\alpha_{1}, \alpha_{2}$ の值は，材料の異方性の程度によって 異なり， $G_{x y}, \nu_{x}$ 等が同じ材料については, $E_{x} / E_{y}$ の 值が 1 に近いほど，その差が小さくなる．したがって $E_{x} / E_{y}$ の值が 1 に近い材料では, $\alpha_{1}^{0} \alpha_{2}^{l}, \alpha_{1}^{2} \alpha_{2}^{0}$ の值と $\alpha_{1}^{1} \alpha_{2}^{1}$ の值とがほほ同じオーダとなる.このことょり $E_{x} / E_{y}$ の值が 1 に近い材料では $U_{11}$, あるいは, さら にU $U_{i j(i+j>2)}$ をも考慮しなくてはならない.しかし，当 節ねじ継手の対象となっている瀻維強化材料等では $E_{x} / E_{y}$ の值が 1 より離れている場合が多く， $\alpha_{1}^{1} \alpha_{2}^{1}$ の オーダは $\alpha_{1}^{2} \alpha_{2}^{0}$ 等に比し小さくなる.したがって $U_{11}$
の影響は小さくなると考元られる。

このように，異方性の状況によっては，取るべき項 数を多くする必要があり，それによって応力関数の誘 導も難しくなるのが本手法の欠点でもある。しかし， 従来, 直交異方性材の応力分布については, 理論的に も把握するすべがなかったことを考慮すると，概略值 といえど解析できることは，当該分野に資すると考え ている.

（3）実際のねじでは，フランク面に沿って分布荷 重が作用するが，ここでは，有効径に相当する点に集 中荷重として作用させた。したがって, 有効径近傍で は, 集中荷重による影響が現れているが（弾性学の諸 問題でも荷重集中点は特異点となり，応力無限大とな る), 谷底では，その影響は少なくなり，最大応力の値 等の評価に差支えない．これは，等方性の場合の理論 的扱い，光弾性実験による扱いにも共通している。し かし，ご指摘のように，ねじ結合の状態によっては， 荷重点が，㸚じ谷底近くなったりねじ山先端近くなっ りすることもある。このうち，荷重点が，㸚じ谷底近 くになると，荷重点近傍の応力の乱れの影響が谷底の 応力分布に影響を及ほし，分底の応力分布を正確には 評価し得なくなる。 